\title{
APORTACION AL ESTUDIO DE LOS HELECHOS DE LA CUENCA EXTREMEÑA DEL GUADIANA
}

\author{
J.L. PEREZ CHISCANO (*)
}

\section{RESUMEN:}

Se citan los helechos conocidos en la cuenca del río Guadiana (Extremadura). Se dan nuevos táxones para la flora extremeña y se citan nuevas localidades para varias especies.

\section{SUMMARY:}

The ferns of Guadiana's Bassin (Extremadura, Spain) are listed in this paper, together with some new fern taxa. Also the author gives new localities and commentaires.

\section{INTRODUCCION}

Las especies mencionadas están en la cuenca extremeña del río Guadiana que comprende la mayor parte de la provincia de Badajoz, el borde sur de la de Cáceres y los occidentales de Ciudad Real y Córdoba. Esta publicación es sólo un avance del estudio que estamos haciendo sobre corología, ecología y sistemática de este interesante grupo de pteridofitas. Los trabajos precedentes sobre los helechos extremeños han sido hechos por Rivas Goday (1964), Ladero (1970), Rivas Martínez y Ladero (1972), Rivas Goday y Ladero (1973) y Pérez Chiscano (1976).

Los helechos se encuentran en los siguientes biótopos. Crestas rocosas de sierras que en su mayoría son cuarcitas ordovícicas, granitos hercínicos y calizas cámbricas. Bosques montanos de robles, castaños, quejigos y alcornoques, preferentemente en las laderas de umbría. Bosquetes riparios de alisos, chopos y fresnos. Raras veces en los habitat húmedos de llanos. Todo ello dentro de un fitoclima de tipo mediterráneo, pero a veces con marcada influencia atlántica, de aquí la presencia de helechos de área oceánica. 


\section{LISTA DE ESPECIES}

Adiantus capillus-veneris $\mathrm{L}$.

Anogramma leptophylla (L.) Link

Asplenium billotii Schulz

Asplenium onopteris L.

Asplenium trichomanes L.

Athyrim filix-femina (L.) Roth

Azolla caroliniana Willd.

Blechmum spicant (L.) Roth

Ceterach officinarum $\mathrm{DC}$.

Cheilamthes duriensis Mend. \& Vass.

Cheilanthes hispanica Mett.

Cheilamhes maderensis Lowe
Cheilathes preridioides (Reich.) C. Chr. Cheilanthes vellea (Ait.) Muell.

Cistosteris fragilis (L.) Bernh.

Dropteris pseludomas (Woll.) Holub \& Pouzar

Marsilea strigosa Willd.

Ophioglossum lusitanicum L.

Osmunda resalis L.

Pollpodium australe Fè

Polipodium interjectum Shivas

Polystichum setiferum (Forskal) Woynar

Preridium acpuilinum (L.) Kuhn

NUEVOS TAXONES PARA LA FLORA Y NUEVAS LOCALIDADES PARA OTRAS ESPECIES YA CONOCIDAS.

Se dan las localidades con las coordenadas UTM de las euadriculas de $100 \mathrm{kms}$ cuadrados, algunos datos ecológicos y los testimonios de herbario (MAF y el nuestro particu$\operatorname{lar} \mathrm{P}(\mathrm{H})$.

Adiantum capillus-veneris L., Sp. Pl. (1753)

Rivas Goday le cita por vez primera cerca de Guadalupe como caracteristica territorial de la as. Lunulario-Selaginelletum. En 1975 la vimos en fisuras rezumantes de calizas serva de Jerez de los Caballeros (PC-94) (MAF-96232, Bote, Ladero \& Pérez Chiscano). Esta comarca tiene una de las precipitaciones más altas de la cueinca, de 1.000 a $1.200 \mathrm{~mm}$. anuales, factor importante para la presencia de este helecho que por otra parte debe ser muy escaso en Extremadura.

Athyrium filix-femina (L.) Roth Tenr. Fl. Germ. 3 (1): 65 (1799)

Es frecuente en los rios y arroyos del Macizo de las Villuercas (Al. Osmundo-Alnion). Damos como nueva localidad un barranco umbroso en la sierra de Valdecaballeros (UJ14) (MAF-84645, Pérez Chiscano) (PCH-718, Pérez Chiscano).

Azolla caroliniana Willd., Sp. Pl. 5 (1): 541 (1810)

Detectada hace poco tiempo (en 1974, seguramente invadió bastante antes), se ha extendido por las aguas estancadas, ríos y arrozales de las vegas. Al cubrir la superficie de los grandes charcos naturales impide la penetración de la luz con el consiguiente empobrecipiento de las plantas sumergidas (Potametea) y el deterioro de la biocenosis que de cllas depende.

Creemos que se trata de la especie indicada por sus márgenes de frondes estrechos y tener los gloquidios de las másulas septados. (PCH-933, Pérez Chiscano, río Ruecas) (PCH-2444, P. Chiscano, río Guadiana en Medellín). 
Blechnum spicant (L.) Roht, Useri Ann. Bor. 10: 56 (1794)

Encontrado en área finícola en un arroyo umbroso de Valdecaballeros (UJ-14) (MAF84646, Pérez Chiscano) (PCH-715 y 2173, Pérez Chiscano).

Cheilanthes duriensis Mend. \& Vasc., Anais Inst. Vinho Porto, 15 (4): 47 (1956)

(Ch. fragrans (L. til.) Swartz var. negleta Azn.) (Ch. x duriensis Mend. \& Vasc., 1. c.) (Ch. hispanica $\mathrm{x}$ Ch. pteridioides) (Ch. corsica Reichst. \& Vid.) (Ch. tinaei Todaro).

Le vimos por primera vez en la Sierra del Pedroso (TH-76) (MAF-88642, Pérez Chiscano) y más tarde en numerosas localidades. Se encuentra entre los bloques de piedras sueltas de las pedrizas y en la base de las cuarcitas de cumbre, donde el suelo es más terroso y hay un microclima algo mesofítico, en contraste con la ubicación de Ch. hispanica que ocupa las tisu ras de los peñones en exposición más descubierta y soleada. Hasta ahora le hemos encontrado en Sierra de la Chimenea-Puerto Peña (UJ-03) (PCH-2356, Pérez Chiscano), Sierra de Castuera-Puerto Mejoral (TH-88) (PCH-2352, Pérez Chiscano), Sierra de la Ortiga (TJ-50), riberos pizarroso del río Ardila entre Zafra y Fregenal de la Sierra (QC-14) (PCH-2370, Pérez Chiscano), pizarras del río Sillo, Higuera la Real (QC-02), Sierra de Pinos-Hornachos (QC-57) (PCH-2371), Cuarcitas del castillo de Azagala (PD-84) (PCH-2406, P. Chiscano), Sierra Anarón cerca del río Valdeazogue (UH-38), Peñas Blanàs, Oliva de Mérida (QC-49) (PCH-2348 y 2532, Pérez Chiscano).

Cheilanthes maderensis Lowe, Trans. Cambr. Phil. Soc. 6: 528 (1838)

(Ch. preridioides (Reich.) C. Chr. var. maderensis (Lowe) Chr. (Ch. pteridioides (Reich.) Chr. fma. maderensis (Lowe) Maire \& Weiller).

Rivas Martínez y Ladero (1971) le citan en los riberos soleados de los ríos que vierten al Tajo en Las Villuercas. Le vamos encontrando en varios lugares de la cuenca del Guadiana siendo su habitat preferido debajo de pied ras sueltas y base de peñones cuarcitosos (dentro del fitoslima de Asparago-Rhamnion oleoidis), esto es, microclimas térmico-húmedos. En alyunas sierras coexiste con $C h$. duriensis, pero este último prefiere sitios más umbrosos. Hasta ahora visto en solanas de Castuera-Puerto Mejoral (TH-88) (PCH-259, - Pérez Chiscano) Castillo de Almorchón (UH-08) y riberos del río Matachel (TH-46) (PCH-2373, Pérez Chiscano).

Cheilanthes pteridioides (Reich.) C. Ch., Ind. Fil. 178 (1960) (Ch. fragrans (L.) Schott)

Como planta preferentemente calcicola sólo la hemos visto en las calizas paleozoicas de Sierra Calera, Santa Marta de los Barros (QC-84) (MAF-92646, Pérez Chiscano) (PCH2373, Pérez Chicano) y en Puebla de Reina (TH-43) (PCH-2350, Pérez Chiscano).

Cheilanthes vellea (Ait.) F. Muell., Frag. Phylogr: Austr. 5: 123 (1866)

(Ch. catanensis (Cosen.) H. P. Fuchs) (Notholaena rellea (Aiton) Desv.) (N. lanuginosa (Deff.) Poiret).

La primera cita para Extremadura es de Monserrat Recoder en el anfiteatro romano de Mérida (Rivas Goday \& Ladero, 1973), donde más tarde volvimos a encontrarla (MAF83993, Pérez Chiscano) (PCH-562, Pérez Chiscano). También se en cuentra en las calizas del Cerro Carija en la misma comarca, siempre en solanas y fitoclima de acebuches (as. Asparago-Rhamnetum beturici) (QD-21) (PCH-1957 y 1970. Pérez Chiscano). Otras locali- 
dades en las calizas cámbricas de Sierra Calera cerca de Santa Maria de los Barcos (QC-84) (PCH-1929) asi como en suelos básicos del rio Albarragena (PD-84) (PCH-2405. Pérez Chiscano).

Ophioglossum lusitanicum L., Sp. Pl. 1.063 (1753)

Conocido como escaso, ha resultado ser uno de los helechos más abundantes de la cuenca del Guadiana extremeño. Se encuentra en los pastizales de Poetalia bulbosae de suelos pardos poco profundos sobre pizarras y litosuelos sobre granitos. Es particularmente copioso en La Serena en otoños lluviosos, Villanueva de la Serena (TJ-51), Magacela (TJ-60), La Coronada (TJ-61), Campanario (TJ-70) (MAF-99382, Pérez Chiscano), Orellana la Vieja (TJ-82), Almorchón-Cabeza del Buey (UH-80), Quintana de la Serena (TH66), Capilla (UH-29). También en Herrera del Duque (UJ-13), Castilblanco (UJ-15), San Pedro de Mérida (QD-41) (MAF-83621, Rivas Goday \& Ladero), Valdebotoa (PD-72), Alburqueque (PD-74) (MAF-83620, Rivas Goday \& Ladero) y Campolugar (TJ-64).

Osmunda regalis L., Sp. Pl. 1.065 (1753)

Algo frecuente en los arroyos montanos de Las Villuercas y en río Benazaire cerca de Herrera del Duque (UJ-24) (MAF-75807, Rivas Goday \& Ladero) en las llamadas "islas atlánticas de vegetación" (Rivas Godayaño) y Helechosa de los Montes (UJ-35). También le hemos encontrado en barrancos umbrosos de la sierra de Valdecaballeros (UJ-14) (MAF-89964, Pérez Chiscano) (PCH-716 y 834) como localmente abundante, en orillas del río Ruecas cerca de Logrosán (TJ-85) en avanzadas de las comunidades de Osmundo-A/nion que bajan desde las Villuercas amparadas por la humedad edálica del rio. También en orillas del río Albarragena cerca de Alburquerque (PD-84) en un destiladero umbroso de cuarcitas.

Polypodium australe Fée, Mém. Fam. Foug. 5: 236 (1852)

(P. serratum (Willd.) Sauter, non Aublet).

Opinamos que se trata de ecta especie por tener los siguientes caracteres morfológicos. Escamas del rizoma asi $11 \mathrm{~mm}$. de longitud, soros con paralisos que desaparecen al madurar las esporas y como máximo 9 células en el anillo del esporangio. Se encuentra en las repisas de los roquedos de cumbre con orientación de umbría, tanto en cuarcitas como en calizas. No fisuricola, se asienta entre la capa delgada del estrato muscinal que se instala cobre el escaso suelo que cubre la roca. Esta capa se desprende con facilidad tirando de ellat. Debido a lo poco accesible de estas cornisas nos faltan por recolectar muchos de estos helechos en las sierras de la euenca del Guadiana y en general de Extremadura, pero por lo va visto parece que su localización geográlica es más meridional que P. interjectum, la otra especie del género en el territorio. Los lugares hasta ahora son los siguientes. Cuarcitas del castillo de Almorchón (UH-08) (PCH-2535. Pérez Chiscano), arroyo Friegamuñoz cerca de Cheles (PC-56), sobre cuarcitas. También en cuarcitas en Santo Doningo de Olivenza (PC-68) y sobre calizas cámbricas en la sierra de La Parra (QC-06). En las localidades mencionadas sólo se encuentra esta especie, pero en las cuarcitas de Montfragüe, en el Tajo (QE-51) (MAF-78757, Rivas Goday) está con P. interjectum. En opinión de Rivas Martínez (comunicación personal), pudiera tratarse de $P$. $x$ shivasiae Rothm, hibrido entre ambas especies, cuestión que aún no hemos aclarado. 
Polypodium interjectum Shivas, Jour: Linn. Soc. London (Bot.) 58: 28 (1961)

Como la especie anterior se instala en las cornisas rocosas de umbria. Hasta ahora sólo encontrado en cuarcitas. También en el estrato de musgos sobre la roca. Este sustrato tiene una buena proporción de humus. De un análisis mecánico en muestra procedente del Peñón de Cogolludo (Puebla de Alcocer), encontramos que la proporción húmica y. restos vegetales es del $58,83 \%$ y la parte mineral del $47,17 \%$ (repartida en $32,29 \%$ para la fración areno-limosa y 5,88\% para la arcilla). Las localidades hasta ahora son, sierra de Cañamero en el valle alto del río Ruecas (T J-96), sierra de La Chimenea-Puerto Peña (UJ03 y UJ-13) (PCH-248, Pérez Chiscano), Peñón de Cogolludo (TJ-92) (PCH-2538, Pérez Chiscano), sierra de Peñas B lancas Oliva de Mérida (QC-49) (PCH-2539, Pérez Chiscano) y Puero-Mejoral, Castuera (TH-88) (PCH-2589, Pérez Chiscano).

\section{BIBLIOGRAFIA}

JALAS, J. \& SUOMINEN, J. -1972- Atlas Florae Europaeae, Tomo I, Pteridophyta, Helsinki. LADERO, M. - 1970-Contribución al estudio de la flora y vegetación de la comarca de La Jara, serrania de Ibor y Guadalupe-Villuercas en la Oretana Central. Tesis doctoral. Madrid.

PEREZ CHISCANO, J.L. - 1975- Vegetación a rbórea y arbustiva de las sierras del noreste de la provincia de Badajoz. Tesis doctoral. Madrid.

PEREZ CHISCANO, J.L. -1976- Charnecales y madroñales del noreste de la provincia de Badajoz. Anal. Inst. Bot. Cavanilles 34 (2): 497-519. Madrid.

RIVAS GODAY, S. -1964- Vegetación y flórula de la cuenca extremeña del Guadiana. Dip. Prow. de Badajoz. Madrid.

RIVAS GODAY, S. \& LADERO, M. -1973- Nuevas aportaciones a la flora pacense. Anal. Real Acad. Farmacia 39 (3): 267. Madrid.

RIVAS MARTINEZ, S. -1975- Mapa de la vegetación de la provincia de Avila. Anal. Inst. Bot. Cavanilles 32 (2): 1493-1559, Madrid.

RIVAS MARTINEZ, S. \& LADERO, M. -1971- Los pteridófitos de las Villuercas (Cáceres). Anal. Inst. Bot. Cavanilles 28: 37-61. Madrid.

SAENZ DE RIVAS, C. \& RIVAS MARTINEZ, S. -1979- Revisión del género Cheilanthes (Sinopteridaceae) en España. Lagascalia 8 (2): 215-241. Sevilla.

TUTIN, T.G. \& al. -1964- Flora Europeae, Tomo I. Cambridge University Press. Cambridge. 